\title{
Bedürfnislandschaft älterer Menschen in Österreich
}

\author{
Dorothea Erharter, Elka Xharo
}

ZIMD - Zentrum für Interaktion, Medien \& soziale Diversität

\section{Zusammenfassung}

Im Rahmen eines Forschungsprojekts mit dem ÖIAT und B-NK wurden in einer Cultural Probe Studie die Bedürfnisse älterer, zuhause lebender Menschen (60+) in Österreich erforscht. Die Testpersonen dokumentierten ihre Wünsche, Bedürfnisse und Ärgernisse und lieferten zahlreiche Anknüpfungspunkte für die Entwicklung von mobilen Apps für SeniorInnen. Die dominierenden Themen waren hierbei Unterstützung im Haushalt, Medizinisches, Gartenarbeit, sowie soziale Kontakte und Familie.

\section{$1 \quad$ Einleitung}

Seniorinnen und Senioren haben aktuell die höchste Zuwachsrate bei der Nutzung des Internets und verfügen hinsichtlich der Nutzung von mobilen Endgeräten, wie Smartphones oder Tablet-PCs, über große Wachstumspotenziale. Der Zugang zum Internet bzw. dessen kompetente Nutzung stellt heute einen wichtigen Aspekt gesellschaftlicher Teilhabe (,Social Inclusion") dar. Vor diesem Hintergrund wurde im Projekt MOBISENIORA untersucht, welche spezifischen Anforderungen ältere Nutzerinnen und Nutzer bei der Verwendung mobiler Geräte für die Internetnutzung haben. (vgl. Erharter et al. 2014, Xharo \& Erharter 2014, AmannHechenberger 2015)

Einen Teil des Projekts bildete eine Cultural-Probe Studie, in der das ZIMD die Wünsche, Sehnsüchte und Ärgernisse zuhause lebender Seniorinnen und Senioren in Österreich erforschte. Primäres Ziel der Cultural-Probe Studie war, anhand der Ergebnisse Ideen für Apps für ältere Menschen zu generieren. Durch den besseren Einblick in deren Alltagswelt wird es möglich, Apps zu entwickeln, die auf ihre Bedürfnisse eingehen und ihnen den Anschluss ins digitale Zeitalter erleichtern.

$\mathrm{Zu}$ den Bedürfnissen von Seniorinnen und Senioren gibt es zahlreiche Studien, doch häufig wird mit Einrichtungen für betreutes Wohnen kooperiert. In den „eigenen vier Wänden“ wohnende Seniorinnen und Senioren haben allerdings teilweise andere Bedürfnisse als Menschen 
in Betreuungseinrichtungen. Die von uns entwickelte Bedürfnislandschaft älterer Menschen halten wir aus diesem Grund für innovativ und beispielgebend auch für andere Regionen.

\section{Das Setting}

\subsection{Die „Ideentagebücher“}

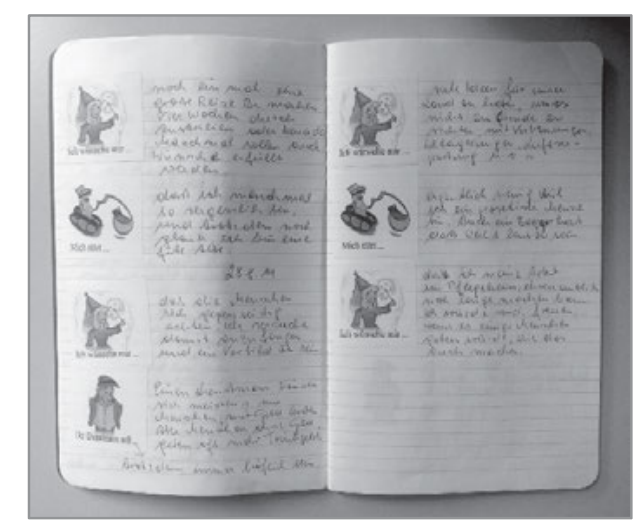

Abbildung 1: Ideentagebuch einer Testperson

In der vorliegenden Studie ging es darum, ein für die Testpersonen einfaches und vertrautes Setting zu entwickeln. Daher kam letztlich ein Notizheft mit Stickern zum Einsatz. Die Testpersonen sollten damit ein „Ideentagebuch“ führen (vgl. Abb. 1). Anhand von drei Metaphern notierten die Testpersonen Wünsche und Interessen, die ihnen im Verlauf ihres Alltags in den Sinn kamen. Es konnten drei unterschiedliche Sticker geklebt werden, dabei stand die „Fee“ für Sehnsüchte und Wünsche, der „Dienstmann“ für Unterstützung und Service und der „Bagger" für Ärgernisse und Hürden (vgl. Abb. 1). Jeweils sieben Tage lang und jeweils in drei verschiedenen Jahreszeiten vermerkten die zwölf Testpersonen Wünsche, Sehnsüchte und Ärgernisse. Die 12 Testpersonen waren (6 Männer, 6 Frauen) zwischen 60 und 75 Jahre alt und bereits in Pension. Um eine möglichst hohe Diversität zu erreichen, wurden die Cultural Probes in einer großstädtischen, einer kleinstädtischen und einer ländlichen Region durchgeführt.

Die resultierenden 960 Tagebucheinträge aus den Cultural Probes wurden digital erfasst und von drei Personen unabhängig voneinander 50 verschiedenen Kategorien zugeordnet. 


\section{Ergebnisse}

Insgesamt können folgende wiederkehrenden Themenbereich festgehalten werden: Oftmals werden diverse Unterstützungstätigkeiten für Arbeiten im Haushalt gewünscht, meist in $\mathrm{Zu}-$ sammenhang mit aufwändigeren oder beschwerlichen Tätigkeiten, wie Keller aufräumen oder Vorhänge abnehmen. Medizin und Gesundheit sind wichtige Themen, über die sich ältere Menschen häufig informieren wollen. Ein großer Bereich der Einträge beschäftigte sich auBerdem mit Besorgungen und Wegen, wie auch Gartenarbeit. Viele Einträge behandelten auch abstrakte Themen wie dem Wunsch nach einer ,besseren Welt“" oder Ärger über andere Menschen und Institutionen. Eines der dominierenden Themen waren Zwischenmenschliches und Kontakt zu Familie und Freundinnen und Freunden. Viele ältere Menschen wünschten sich z.B. auch Begleitung zu Aktivitäten.

Zwischen den Geschlechtern gab es nur wenig Unterschiede. Bei den weiblichen Testpersonen wurden etwas häufiger die Kategorien „Wünsche für andere“, „Ärger/Wünsche an sich selbst“, „Gartenarbeit“, sowie „Freunde/Soziales“ verwendet. Bei den Männern gab es bei den Kategorien „Besorgungen/Wege“, „EDV-Probleme“, „Politik“ und „Medizinische Probleme“ einen leichten Überhang.

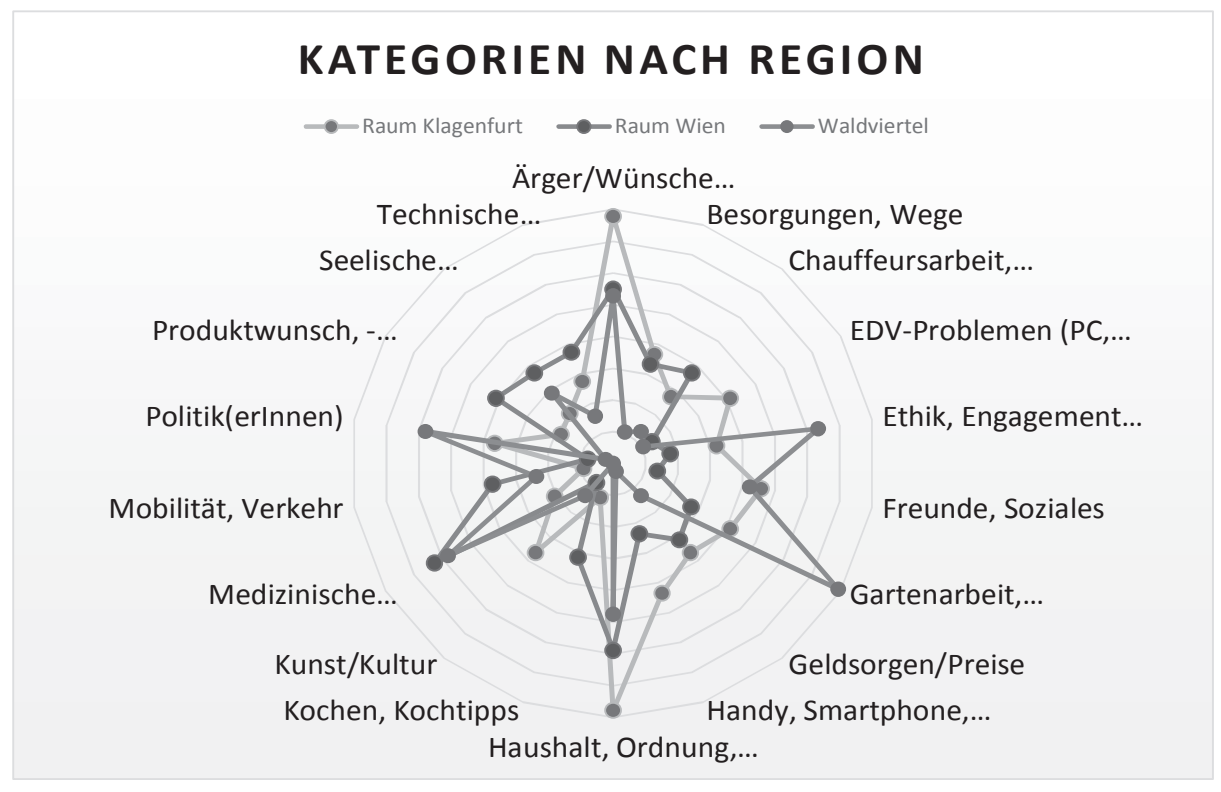

Abbildung 2: Kategorien nach Regionen

Die regionalen Unterschiede waren etwas größer als die Gender-Unterschiede. Vor allem bei den Themen „Politik“, „Gartenarbeit“, „Wetter“, „Ethik/Engagement“" und „Technische Unterstützung“" (vgl. Abb. 2). 


\section{Ausblick}

Die Ergebnisse der „Ideentagebücher“ sollen nun webbasiert interaktiv umgesetzt werden, so dass auch die einzelnen Tagebucheinträge, die die Lebenswelten der Seniorinnen sehr anschaulich machen, (anonym) abrufbar sind, und nach Geschlecht, Region, Jahreszeit bzw. Metapher (Fee, Dienstmann, Bagger) gefiltert werden kann.

Weiters wird auf Basis der im Forschungsprojekte bereits begonnenen Liste an App-Ideen eine App-Plattform für ältere Menschen entwickelt, auf der redaktionell ausgewählte Apps zur Verfügung gestellt und bewertet werden können.

\section{$5 \quad$ Schlussteil}

\section{Literaturverzeichnis}

Amann-Hechenberger, B., Buchegger, B., Erharter, D., Felmer, V., Fitz, B., Jungwirth, B., Kettinger, M., Schwarz, S., Knoll, B., Schwaninger, T. \& Xharo, E. (2015): Tablet \& Smartphone: Seniorinnen und Senioren in der mobilen digitalen Welt. Forschungsbericht zum Projekt „mobi.senior.A “. Unter Mitarbeit von Kraler, D., Landl, A. H., Olsacher E. \& Spreitzer, G.. Wien. Online verfügbar unter http:// forschungsbericht.mobiseniora.at/forschungsbericht.pdf. Zuletzt abgerufen am 5.6.2015

Erharter, D., Jungwirth, B., Schwarz, S., Knoll, B., Posch, P. \& Xharo, E. (2014): Smartphones, Tablets, App für Seniorinnen und Senioren. In: Guido Kemper (Hg.): Assistenztechnik für betreutes Wohnen: Beiträge zum Usability Day XII. Pabst Science Publishers.

Sanders, Elizabeth; SonicRim, Ltd (1999): Postdesign and Participatory Culture. In: Useful and Critical: The Position of Research in Design. Online verfügbar unter http://www.maketools.com/articles-papers/PostdesignandParticipatoryCulture_Sanders_99.pdf, zuletzt geprüft am 23.01.2015.

Xharo, Elka; Erharter, Dorothea (2014): SeniorInnen und Usability bei Smartphones und Tablets. In: Markus Seidl und Grischa Schmiedl (Hg.): Forum Medientechnik - Next Generation, New Ideas. Beiträge der Tagung 2014 an der Fachhochschule St. Pölten. Glückstadt: Werner Hülsbusch, S. 255268. Online verfügbar unter https://phaidra.fhstp.ac.at/detail_object/o:104?SID=6225\&actPage $=1 \&$ type $=$ listview\&sortfield=fgs.createdDate,STRING\&sortreverse $=0$, zuletzt geprüft am 16.04.2015.

\section{Kontaktinformationen}

Dorothea Erharter, ZIMD, 1180 Wien, Währinger Straße 81/12. d.e@zimd.at.+43-699-11369902. 